\title{
PÊNFIGOS NA AMAZÔNIA: ASPECTOS CLÍNICOS, EPIDEMIOLÓGICOS E TERAPÊUTICOS DE PACIENTES ATENDIDOS EM SERVIÇO DE REFERÊNCIA
}

\author{
PEMPHIGUS IN AMAZON: CLINICAL, EPIDEMIOLOGICAL AND \\ THERAPEUTIC ASPECTS OF PATIENTS ATTENDED AT A REFERENCE SERVICE \\ Marcela Coutinho Alves, Carla Andréa Avelar Pires, Carla dos Santos Lima, Juliana Leão de \\ Souza Duque, Caren dos Santos Lima \\ Universidade do Estado do Pará
}

\begin{abstract}
The term "pemphigus" is used to describe a group of autoimmune bullous diseases involving skin and mucosal surfaces. It is characterized histologically by intraepidermal cleavage and IgG deposition on the keratinocytes surface. Pemphigus vulgaris (PV) and Pemphigus foliaceus (PF) are the main types of pemphigus. This study aimed to identify the demographic, clinical and therapeutic aspects of pemphigus. A retrospective analysis of medical records was conducted in the archive sector of the Dermatology Outpatient Clinic of the University of the State of Pará (UEPA) from January 2007 to January 2017. We found 19 patients with pemphigus were identified, 11 of whom had PV and 8 had PF. Both types of pemphigus were more common in women (57.89\%). The age group most affected by PV was 22 to 59 years old (81.82\%), whereas by in PF it was the age group of 60 years old (62.5\%). The main area affected in PV was upper limbs (20.69\%) and in PF, the main one wasface $(21.43 \%)$. Prednisone was the most prevalent drug regimen (42.87\%). Epidemiological data on the disease are still scarce, especially in northern Brazil. There is no consensus regarding the treatment and the choice must be based on the particularities of each case.
\end{abstract}

Keywords: Pemphigus; Autoimmunity; Vesiculobullous; Epidemiology
Resumo

O termo "pênfigo" é usado para descrever um grupo de doenças bolhosas autoimunes que comprometem a pele $e$ as mucosas. É histologicamente caracterizado pela clivagem intraepidérmica e deposição de IgG na superfície dos ceratinócitos. Pênfigo vulgar e pênfigo foliáceo são as principais formas clínicas. Essa pesquisa objetivou identificar os aspectos demográficos, clínicos e terapêuticos dos pacientes com diagnóstico de pênfigo. Para isso, foi realizada análise retrospectiva de prontuários médicos nos arquivos do ambulatório de Dermatologia da Universidade do Estado do Pará de janeiro de 2007 a janeiro de 2017. Foram identificados 19 pacientes com diagnóstico de pênfigo, dos quais 11 tinham pênfigo vulgar, e 8, pênfigo foliáceo, sendo ambas as formas clínicas mais prevalentes em mulheres (57,89\%). A faixa etária mais acometida por pênfigo vulgar foi de 22 a 59 anos (81,82\%), enquanto por pênfigo foliáceo foram os maiores de 60 anos (62,5\%). A principal área afetada por Pênfigo vulgar foram os membros superiores (20,69\%), e por pênfigo foliáceo, a face (21,43\%). Prednisona foi o tratamento mais utilizado (42,87\%). Observou-se que os dados epidemiológicos referentes a essa patologia ainda são escassos, especialmente na região Norte do Brasil. Ainda não há consenso referente ao tratamento, e a escolha de medicações deve ser baseada nas particularidades de cada caso.

Palavras-chave: Pênfigo; Autoimunidade; Dermatopatias Vesiculobolhosas; Epidemiologia. 


\section{Introdução}

O termo "pênfigo" é utilizado para descrever um grupo de doenças bolhosas autoimunes que envolve pele e mucosas. É caracterizado histologicamente pela formação de bolha acantolítica intraepidérmica e depósito de IgG na superfície dos queratinócitos. A deposição de autoanticorpos leva à acantólise, que consiste na perda da adesão entre as células epiteliais de Malphigi. ${ }^{1}$ Estudos demonstram que os autoanticorpos circulantes e ligados à pele nos pênfigos pertencem, sobretudo, a diferentes classes de IgG, principalmente IgG1 e IgG4. ${ }^{2} \mathrm{O}$ pênfigo vulgar (PV) e o pênfigo foliáceo (PF) são as principais variantes histológicas da doença.

O PV é a forma mais frequente na população mundial e a mais severa. Neste tipo, a acantólise ocorre no estrato espinhoso suprabasal e caracteriza-se pela presença de anticorpos antidesmogleína 3 (anti-dsg3) na forma mucosa e de anti-dsg1 e 3 na forma mucocutânea. Possui distribuição mundial, ocorre de forma semelhante em ambos os sexos e pode acometer qualquer faixa etária, tendo o seu pico de incidência entre a quarta e a sexta década de vida. ${ }^{3}$ O quadro clínico inicia-se com lesões orais na forma de lesões ou úlceras aftosas e posteriormente afeta a pele, com o aparecimento de vesículas e bolhas flácidas com conteúdo claro ou turvo. ${ }^{1}$ A forma vegetante é uma variante do $\mathrm{PV}$.

No PF, a acantólise ocorre na camada granulosa subcórnea, e apresenta apenas antidsg1. Possui duas formas de apresentação que se diferenciam por suas epidemiologias: pênfigo esporádico (também conhecido como não endêmico ou pênfigo de (azenave), o qual tem distribuição mundial, e o pênfigo endêmico (também chamado de fogo selvagem), presente em países como Brasil, Colômbia, Peru, África do Sul e Tunísia. ${ }^{3}$

No Brasil, o fogo selvagem é encontrado no Centro-Oeste e no Sudeste e geralmente incide em adultos jovens e crianças moradoras de áreas rurais. Há pesquisas que associam a picada pelo mosquito Similium como o fator desencadeador da resposta autoimune presente na doença. O quadro clínico é caracterizado por bolhas superficiais que se rompem facilmente, além de áreas eritematosas, escamas e crostas, poupando mucosas. ${ }^{1,4}$
Há também outros tipos mais raros de pênfigo, entre eles: pênfigo herpetiforme (quadro semelhante à dermatite herpetiforme, porém com achados histológicos compatíveis com pênfigo), pênfigo por IgA (erupção vésicopustulosa, infiltrado neutrofílico, acantólise e IgA circulantes), pênfigo paraneoplásico (lesão cutâneo-mucosa associada a uma neoplasia), pênfigo por IgA/IgG (marcado por depósitos de IgA, IgG e por vezes C3) e pênfigo induzido por drogas (como por exemplo, penicilinamina, captopril, rifampicina e outros medicamentos que também possuem o grupo sulfidril, presente nas demogleínas 1 e 3). ${ }^{5}$

O diagnóstico de pênfigo é realizado pela história clínica e exame físico e confirmado por biópsia de pele, podendo ser necessário completa-la com imunofluorescência direta ou indireta. ${ }^{6} \mathrm{O}$ tratamento é realizado por meio de corticosteróides sistêmicos (prednisona 1-2 $\mathrm{mg} / \mathrm{kg} / \mathrm{peso}$ ) e imunossupressores por longos períodos (azatioprina, ciclofosfamida, metrotexate), por vezes em regime de internação devido à gravidade da doença. ${ }^{1,6,7}$ Esses pacientes são mais suscetíveis a infecções secundárias das lesões, podendo, inclusive, evoluir para sepse e óbito. Ressalta-se também a possibilidade de desenvolvimento de efeitos colaterais causados pela terapêutica adotada (osteoporose, catarata, glaucoma, diabetes tipo 2, úlceras gástricas, anemia, entre outras), justificando a necessidade de acompanhamento frequente por equipe multidisciplinar. ${ }^{8}$

Este estudo tem como objetivo identificar os aspectos demográficos, clínicos e terapêuticos do pênfigo em um centro de referência em Dermatologia, no período de janeiro de 2007 a janeiro de 2017.

\section{Metodologia}

O presente estudo caracteriza-se como observacional, transversal, descritivo, retrospectivo e foi realizado através da análise de prontuários de paciente atendidos no serviço de Dermatologia da Universidade do Estado do Pará (UEPA), um centro de referência na região Norte. Foram incluídos prontuários de todos os pacientes portadores de pênfigo atendidos neste ambulatório no período de janeiro de 2007 a janeiro de 2017. Foram analisados aspectos demográficos, clínicos e terapêuticos. Este trabalho foi realizado após aprovação de Comitê de Ética em Pesquisa (Parecer no 1.965.321).

A amostra foi avaliada por estatística descritiva, utilizando medidas de tendência central (média aritmética), variância (desvio 
padrão) e frequência (absoluta e relativa). Para avaliação entre os casos de pênfigo vulgar e foliáceo foi utilizado o Teste G. Toda a inferência estatística foi calculada utilizando o software BioEstat 5.4, adotando p-valor significativo $\leq 0.05$.

\section{Resultados}

Durante o período estudado, foram identificados 19 pacientes com pênfigo, sendo 11 portadores de PV e 8 de PF. Não foram encontradas diferenças estatisticamente significativas entre pacientes com PV e PF nos quesitos gênero, faixa etária e procedência. Em

TAB畐 1: Aspectos demográficos e clínicos de pacientes diagnosticados com pênfigo entre 2007 e 2017.

\begin{tabular}{|c|c|c|c|c|c|c|c|}
\hline \multirow[t]{2}{*}{ Variáveis } & \multicolumn{2}{|c|}{ Vulgar } & \multicolumn{2}{|c|}{ Foliáceo } & \multicolumn{2}{|c|}{ Total } & \multirow[t]{2}{*}{ p-valor } \\
\hline & $\mathrm{n}$ & $\%$ & $\mathrm{n}$ & $\%$ & $\mathrm{n}$ & $\%$ & \\
\hline \multicolumn{8}{|l|}{ Gênero } \\
\hline Masculino & 4 & 36,36 & 4 & 50 & 8 & 42,11 & 0,9015 \\
\hline Feminino & 7 & 63,64 & 4 & 50 & 11 & 57,89 & \\
\hline \multicolumn{8}{|l|}{ Faixa etária } \\
\hline 22-59 anos & 9 & 81,82 & 3 & 37,5 & 12 & 63,16 & 0,1334 \\
\hline$>60$ anos & 2 & 18,18 & 5 & 62,5 & 7 & 36,84 & \\
\hline \multicolumn{8}{|l|}{ Região de origem } \\
\hline Área metropolitana & 8 & 72,73 & 6 & 75 & 14 & 73,68 & 0,6782 \\
\hline Outras & 3 & 27,27 & 2 & 25 & 5 & 26,32 & \\
\hline \multicolumn{8}{|c|}{$\begin{array}{l}\text { Tempo dos primeiros } \\
\text { sinais/ sintomas para } \\
\text { o diagnóstico }\end{array}$} \\
\hline 1 mês < 1 ano & 8 & 72,73 & 2 & 25 & 10 & 52,63 & 0,1456 \\
\hline$\geq 1$ ano & 2 & 18,18 & 4 & 50 & 6 & 31,58 & \\
\hline Desconhecido & 1 & 9,09 & 2 & 25 & 3 & 15,79 & \\
\hline Total & 11 & 100.000 & 8 & 100.000 & 19 & & \\
\hline
\end{tabular}

Em pacientes com $\mathrm{PV}$ a apresentação clínica mais frequente foi a ocorrência de exulceração/crostas/hipercromia/bolhas

(64,29\%). Já em pacientes com PF tanto a forma exulceração/crostas/hipercromia como a forma exulceração/crostas/hipercromia/bolhas tiveram a mesma frequência (40\%).

A distribuição de áreas acometidas apresentou diferenças estatisticamente relevantes entre os dois grupos $(p=0,0066)$. No PV as principais áreas mais afetadas foram membros superiores $(20,69 \%)$, cavidade oral $(20,69 \%)$ e dorso $(17,25 \%)$. No PF, as principais regiões atingidas foram face $(21,43 \%)$, membros superiores $(17,86 \%)$, couro cabeludo $(17,86 \%)$ e tronco $(17,86 \%)$.
TABELA 2: Aspectos clínicos de pacientes diagnosticados com pênfigo entre 2007 e 2017

\begin{tabular}{lccccccc}
\hline Variáveis & \multicolumn{2}{l}{ Vulgar } & \multicolumn{2}{l}{ Foliáceo } & & Total & p-valor \\
Apresentação clínica & & & & & & & \\
\hline & - & - & - & - & - & - & 1.0000 \\
Exulceração apenas & - & - & 4 & 40 & 4 & 16,66 & \\
$\begin{array}{l}\text { Exulceração/crosta/ } \\
\text { hipercromia }\end{array}$ & & & & & & & \\
Exulceração/crosta/ & 9 & 64,29 & 4 & 40 & 13 & 54,17 & \\
hipercromia/bolhas & & & & & & & \\
\hline Áreas afetadas & & & & & & & \\
\hline Face & 3 & 10,34 & 6 & 21,43 & 9 & 15,79 & 0,0066 \\
Abdome & 3 & 10,34 & 2 & 7,14 & 5 & 8,77 & \\
Membros superiores & 6 & 20,69 & 5 & 17,86 & 11 & 19,30 & \\
Membros inferiores & 2 & 6,90 & 3 & 10,71 & 5 & 8,77 & \\
Cavidade oral & 6 & 20,69 & 1 & 3,57 & 7 & 12,28 & \\
Dorso & 5 & 17,25 & - & - & 5 & 8,77 & \\
Couro cabeludo & 1 & 3,45 & 5 & 17,86 & 6 & 10,53 & \\
Tórax & 3 & 10,34 & 1 & 3,57 & 4 & 7,02 & \\
Tronco & - & - & 5 & 17,86 & 5 & 8,77 & \\
\hline Total & 11 & 100,00 & 8 & 100,00 & 19 & 100,00 & \\
\hline
\end{tabular}

relação ao sexo, ambos os tipos de pênfigo foram mais comuns em mulheres (57,89\%). A faixa etária mais acometida por PV foi a de 22 a 59 anos $(81,82 \%)$ enquanto que por PF foi a de pacientes a partir de 60 anos $(62,5 \%)$. Entre os prontuários avaliados, a maioria dos pacientes era procedente da Região Metropolitana de Belém, sendo apenas $26.32 \%$ do interior do estado.

A adesão ao tratamento foi acima de $75 \%$ em ambos os tipos de pênfigo, resultando em 78,95\% dos pacientes aderiram ao tratamento. Apenas 3 pacientes com PV $(27,27 \%)$ e 1 paciente com PF $(12,5 \%)$ foram tratados com monoterapia. Diversos

\begin{tabular}{|c|c|c|c|c|c|c|c|}
\hline $\begin{array}{l}\text { Variáveis } \\
\text { Esquema terapêutico }\end{array}$ & \multicolumn{2}{|c|}{ Vulgar } & \multicolumn{2}{|c|}{ Foliáceo } & \multicolumn{2}{|c|}{ Total } & p-valo \\
\hline Isolado & 3 & 27,27 & 1 & 12,5 & 4 & 21,05 & 0,8330 \\
\hline Combinação & 8 & 72,73 & 7 & 87,50 & 15 & 78,95 & \\
\hline Total & 11 & 100,00 & 8 & 100,00 & 19 & 100,00 & \\
\hline \multicolumn{8}{|l|}{ Drogas } \\
\hline Prednisona & 11 & 44,00 & 7 & 46,66 & 18 & 45,00 & 0,4867 \\
\hline Dapsona & 2 & 8,00 & 3 & 20,00 & 5 & 12,50 & \\
\hline Methotrexate & 1 & 4,00 & 2 & 13,33 & 3 & 7,50 & \\
\hline Azatioprina & 2 & 8,00 & - & - & 2 & 5,00 & \\
\hline Corticoide tópico & 4 & 16,00 & 1 & 6,67 & 5 & 12,5 & \\
\hline Corticoide sistêmico & 3 & 12,00 & - & - & 3 & 7,50 & \\
\hline Tacrolimus & 1 & 4,00 & 1 & 6,67 & 2 & 5,00 & \\
\hline Rituximabe & 1 & 4,00 & 1 & 6,67 & 2 & 5,00 & \\
\hline Total & 25 & 100,00 & 15 & 100,00 & 40 & 100,00 & \\
\hline \multicolumn{8}{|c|}{ Tratamento não especifico } \\
\hline Sim & 4 & 36,36 & 2 & 25,00 & 6 & 31,58 & 0,9790 \\
\hline Não & 7 & 63,64 & 6 & 75,00 & 13 & 68,42 & \\
\hline Total & 11 & 100,00 & 8 & 100,00 & 19 & 100,00 & \\
\hline \multicolumn{8}{|c|}{ Aderência ao tratamento } \\
\hline Sim & 9 & 81,82 & 6 & 75,00 & 15 & 78,95 & 0,8330 \\
\hline Não & 2 & 18,18 & 2 & 25,00 & 4 & 21,05 & \\
\hline Total & 11 & 100,00 & 8 & 100,00 & 19 & 100,00 & \\
\hline
\end{tabular}

esquemas terapêuticos foram usados, sendo a Prednisona a droga mais prevalente (42. 87\%), como mostrado na Tabela 3. Medicações 
sintomáticas, antiparasitários, antifúngicos e antibióticos também foram utilizados para tratar infecções e comorbidades que ocorreram durante o acompanhamento. Todas as condições foram tratadas e não houve nenhuma evolução para óbito.

$$
\text { Os efeitos adversos ao tratamento foram }
$$

TABELA 4: Efeitos adversos ao tratamento em pacientes diagnosticados com pênfigo entre 2007 e 2017.

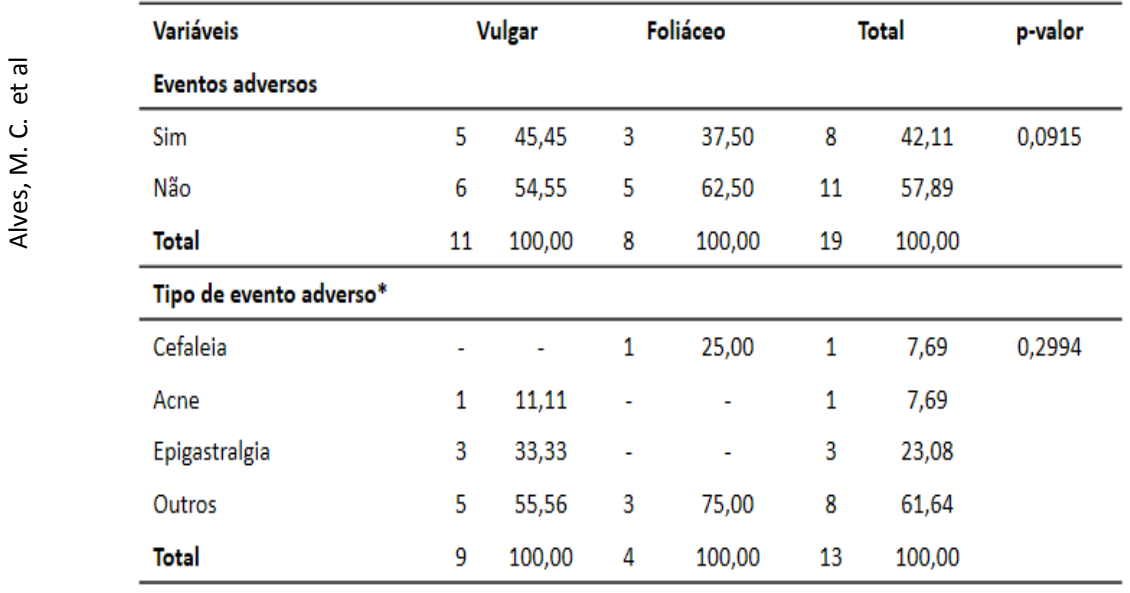

${ }^{*} 0$ mesmo paciente pode ter recebido mais de uma droga

registrados em 8 pacientes $(42,11 \%)$ sendo 5 com PV $(45,5 \%)$ e $3(37,50 \%)$ com PF. Efeitos adversos descritos como "outros" foram os mais relatados $(61,64 \%)$, entre eles estão presentes diarreia, pirose e edema. Epigastralgia foi relatada por 3 pacientes $(23,08 \%)$, dos quais todos eram portadores de PV.

Não houve diferença estatística entre PV e PF no aparecimento de comorbidades $(p=0,2064)$. Nos prontuários avaliados 8 pacientes com PV $(78,73 \%)$ e 6 pacientes com PF (75\%) apresentaram comorbidades. As mais comuns foram Hipertensão Arterial Sistêmica (17,65\%), dermatite seborreica $(17,65 \%)$ e Candidíase $(17,65 \%)$. 


\section{Discussão}

O pênfigo é uma patologia que ainda carece de estudos epidemiológicos, principalmente na Amazônia. Sabe-se que existem diferenças bem estabelecidas entre as distribuições geográficas das principais formas da doença. A incidência de pênfigo vulgar é alta em países como Japão, Índia e Israel, que concentra os maiores índices de PV com 5,3 casos a cada 100 mil habitantes por ano. ${ }^{9,10}$ Isso se deve à predominância do PV entre judeus, em especial aqueles da etnia Ashkenazi. ${ }^{11}$ O pênfigo foliáceo, por sua vez, é uma forma mais rara que se apresenta como endêmica no Peru, Tunísia e em algumas regiões do Brasil. 3,12,13

No entanto, vários estudos vêm demonstrando mudanças importantes no padrão de distribuição geográfica dessas doenças, documentando casos de PV com comportamento endêmico em regiões tipicamente acometidas por PF, como o Centro-Oeste e Sudeste brasileiro. ${ }^{3,14}$

No Pará, a prevalência de pênfigo demonstrou-se baixa ao longo dos anos, bem como na região Amazônica, apesar da escassez de estudos epidemiológicos acerca do tema.,15-18 Entretanto, observou-se que PV foi mais prevalente do que $\mathrm{PF}$, confirmando o padrão documentado em um estudo de 2014, realizado em outro serviço de referência para a doença no Pará. $^{1}$

A distribuição do pênfigo por sexo e idade é bastante variável na literatura mundial. Diversos estudos parecem apontar uma leve predominância pelo sexo feminino, ${ }^{11,14,19}$ a qual é possivelmente explicada pelo aspecto autoimune da doença, tendo em vista que patologias com esse caráter etiológico costumam ser mais comuns entre mulheres, a exemplo do lúpus eritematoso sistêmico, artrite reumatoide, entre outros. Com relação à idade, os dados são ainda mais controversos, com amplas faixas etárias acometidas, que vão de 10 a 39 anos no Peru ${ }^{13}$, até acima de 60 anos no Reino Unido ${ }^{20}$. Estudos brasileiros demonstraram predominância entre a segunda e quinta décadas de vida, em consonância com os resultados obtidos na presente pesquisa. $1,3,14$

Porém, ressalta-se que dentre o grupo de pacientes com PF, a maioria apresentou idade igual ou superior a 60 anos, um dado que reflete a junção de dois fatores de risco para complicação da doença: idade avançada e variante pênfigo foliáceo. A presença desta forma clínica e da não adesão ao tratamento proposto são colocadas como os principais determinantes da morbimortalidade do pênfigo, justificando atenção redobrada no manejo de pacientes que se enquadram nesse grupo. ${ }^{13,19}$

A precocidade do diagnóstico está associada a tratamento com menores doses de corticosteroides, o que diminui reações adversas e melhora o prognóstico. O tempo entre o início dos sintomas e o diagnóstico dos pacientes deste estudo foi semelhante à literatura, sendo a maioria diagnosticada entre o período de 1 mês a 1 ano após os primeiros sintomas, em ambas as formas de doença. ${ }^{1,12}$

As lesões do PV são classicamente bolhas flácidas de conteúdo seroso claro, purulento ou sanguinolento. São facilmente rompidas e geram erosões dolorosas que evoluem para crostas. Podem ser localizadas ou generalizadas e mais frequentemente estão presentes em face, axila e cavidade oral, devido à maior expressão de desmogleína 3 nessas áreas. $^{21,22}$

Já no PF bolhas intactas geralmente não são encontradas, sendo múltiplas erosões crostosas sobre base eritematosa, a sua apresentação clínica mais comum. Geralmente inicia no segmento cefálico com progressão crânio caudal e possui preferência pelo couro cabeludo, face, tórax e costas. O acometimento de mucosas é raro. ${ }^{15,23}$ As características clínicas encontradas neste estudo concordam com estudos presentes na literatura.

Até o presente momento, não há consenso no manejo terapêutico de pacientes portadores de pênfigo, devendo ser consideradas as particularidades de cada caso. No entanto, corticosteroides sistêmicos são considerados a primeira linha de tratamento para reduzir a produção de autoanticorpos ${ }^{7}$, sendo a prednisona a droga mais utilizada. ${ }^{1,4,7}$

A associação com terapia de segunda linha está relacionada a maior eficácia, menores efeitos adversos da corticoterapia e menos recidivas. Podem ser utilizados para este fim medicamentos imunossupressores como azatioprina, metrotexate, ciclofosfamida e dapsona. Neste estudo, apenas 4 pacientes receberam monoterapia com corticoesteroides. A dapsona foi o segundo medicamento mais utilizado. Esta é considerada apropriada como adjuvante durante a fase de manutenção da doença, com eficácia apontada entre $71 \%$ a $83,3 \%$. $^{7,24}$

0 rituximabe foi utilizado em apenas 2 pacientes. É considerado uma terapêutica promissora, porém seu uso é reservado para casos selecionados, com doença refratária ao tratamento convencional. É um medicamento 
imunobiológico de alto custo e com possíveis complicações ainda não totalmente elucidadas ${ }^{24,25}$. O uso de medicamentos não específicos, como sintomáticos, antifúngicos e antiparasitários é associado aos efeitos colaterais resultantes de terapia imunossupressora prolongada. ${ }^{26}$

A adesão ao tratamento é de extrema importância para diminuir a incidência de complicações e a mortalidade. ${ }^{13}$ Como possíveis razões para abandono estão o baixo poder financeiro para uso dos medicamentos a longo prazo, dificuldade de deslocamento até o centro de acompanhamento clínico, e a melhora aparente das lesões. ${ }^{12}$ Nesta pesquisa, a maioria dos pacientes aderiu ao tratamento, refletindo diretamente em baixos índices de complicações e ausência de óbitos.

Os efeitos adversos da corticoterapia, principalmente em uso prolongado, são frequentes e diversos, tais como: cefaleia, irritabilidade, acne, edema, ganho de peso e irritabilidade. $^{26,27} \mathrm{Em}$ pacientes com pênfigo, alterações gastrointestinais são descritas como as mais prevalentes ${ }^{12,28}$ o que concorda com o presente estudo. O médico, portanto, ao propor terapia com corticosteroides deve ter conhecimento sobre suas indicações, exames laboratoriais prévios, seguimento clínico laboratorial, interações medicamentosas e efeitos colaterais, a fim de preservar e/ou melhorar a qualidade de vida dos seus pacientes ${ }^{26}$.

\section{Considerações finais}

Este estudo possibilitou evidenciar características demográficas e clínicas dos pacientes com pênfigo atendidos em centro de referência do Estado do Pará, demonstrando concordância com os dados referentes a idade, sexo, tempo de diagnóstico e apresentação clínica descritos na literatura. Entretanto, dados epidemiológicos sobre a doença ainda são escassos, principalmente no Norte do Brasil, refletindo uma necessidade de mais pesquisas que abranjam esse escopo.

Até o momento, não há consenso em relação ao tratamento da doença e a escolha deve ser baseada nas particularidades de cada caso. A maioria dos esquemas propostos envolvem uso de corticoterapia isolada ou associada a imunossupressores, terapêutica essa também preferencialmente utilizada entre os pacientes estudados. O conhecimento de efeitos adversos relacionados ao uso desses medicamentos, bem como o incentivo a adesão ao tratamento são de fundamental importância para assegurar a boa condução do caso e evitar desfechos indesejados.

\section{Referências}

1. Pires CAA, Viana VB, Araújo FC, Müller SFR, Oliveira MS, Carneiro FRO. Evaluation of cases of pemphigus vulgaris and pemphigus foliaceus from a reference service in Pará state, Brazil. An Bras Dermatol. 2014;89(4): 556-61. URL:

https://www.scielo.br/scielo.php?pid=S036505962014000400556\&script=sci_arttext\&tlng=en 2. Souza SR, Azulay-Abulafia L, Nascimento LV. Validação do índice de comprometimento cutaneomucoso do pênfigo vulgar para a avaliação clínica de pacientes com pênfigo vulgar. An Bras Dermatol. 2011;86(2):284-91. URL: https://www.scielo.br/pdf/abd/v86n2/v86n2a12. pdf

3. Gonçalves GA, Brito MMC, Salathiel AM, Ferraz TS, Alves D, Roselino AMF. Incidência do pênfigo vulgar ultrapassa a do pênfigo foliáceo em região endêmica para pênfigo foliáceo: análise de série histórica de 21 anos. An Bras Dermatol. 2011;86(6):1109-12. URL: https://www.scielo.br/scielo.php?script=sci_artt ext\&pid=S0365-05962011000600007

4. Cortés $C$, Peñaranda $E$, Rodrígues $M$. Fogo selvagem: reporte de dos casos y revisión del tema. Rev Asoc Colomb Dermatol. 2012;20(2): 183-6. URL: https://revistasocolderma.org/sites/default/files/ fogo_selvagem.pdf

5. Porro AM, Caetano LVN, Maehara LSN, Enokihara MMS. Formas não clássicas de pênfigo: pênfigo herpetiforme, pênfigo por IgA, pênfigo paraneoplásico e pênfigo por IgG/IgA. An Bras Dermatol. 2014;89(1): 96-106. URL: http://www.anaisdedermatologia.org.br/detalhe -artigo/101992/Formas-nao-classicas-de-penfigo-penfigo-herpetiforme--penfigo-por-IgA--penfigoparaneoplasico-e-penfigo-por-IgG-IgA-

6. Kayani M, Aslam AM. Bullous pemphigoid and pemphigus vulgaris. BMJ. 2017;357: 1-7. URL: https://www.bmj.com/content/357/bmj.j2169

7. Quaresma MV, Bernardes-Filho F, Hezel J, Peretti MC, Kac BK, Azulay-Abulafia L. Dapsone in the treatment of pemphigus vulgaris: adverse effects and their importance as a corticosteroid sparing. An Bras Dermatol. 2015;90 (3 Suppl 1): S51-4. URL: < https://www.ncbi.nlm.nih.gov/pmc/articles/PMC 4540507/> 
8. Sociedade Brasileira de Dermatologia. Portal da Sociedade Brasileira de Dermatologia. Pênfigo, 2016. [Acesso em 12 abr 2016]. Disponível em http://www.sbd.org.br/doencas/penfigo/.

9. Alpsoy E, Akman-Karakas A, Uzun S. Geographic variations in epidemiology of two autoimmune bullous diseases: pemphigus and bullous pemphigoid. Arch Dermatol Res 2015;307: 291-8. URL: < https://pubmed.ncbi.nlm.nih.gov/25589418/>

10. Femiano F. Pemphigus vulgaris: recent advances in our understanding of its pathogenesis. Minerva Stomatol. 2007;56: 21523. URL: https://pubmed.ncbi.nlm.nih.gov/17452959/>

11. Baum S, Astman N, Berco E, Solomon M, Trau H, Barzilai A. Epidemiological data of 290 pemphigus vulgaris patients: a 29-year retrospective study. Eur J Dermatol 2016; 26(4): 382-7. URL:

https://pubmed.ncbi.nlm.nih.gov/27300747/>

12. Rojas-Plasencia $\mathrm{P}$, Vásquez-Núñez $\mathrm{K}$. Características epidemiológicas, clínicas y supervivencia de pacientes con diagnóstico de pénfigo atendidos en el Hospital Regional Docente de Trujillo. Período 1994-2006. Rev Soc Peruana de Dermatología. 2011;21(4): 148-53. URL:

http://sisbib.unmsm.edu.pe/bvrevistas/dermatol ogia/v21_n4/pdf/a02v21n4.pdf>

13. Muñoz R, Chacon GR, Galarza C, Gutierrez EL, Smith ME, Ortega-Loayza AG. Endemic pemphigus in the Peruvian Amazon: epidemiology and risk factors for the development of complications during treatment. An Bras Dermatol. 2012;87(6): 838-845. URL: < https://pubmed.ncbi.nlm.nih.gov/23197201/>

14. Celere BS, Vernal S, Brochado MJF, Segura-Muñoz SI, Roselino AM. Geographical foci and epidemiological changes of pemphigus vulgaris in four decades in Southeastern Brazil. Int J Dermatol. 2017. URL: < https://pubmed.ncbi.nlm.nih.gov/28737218/>

15. Pinheiro FG, Franco AMR. Pênfigo foliáceo endêmico (fogo selvagem) em indígena Yonomami no município de São Gabriel da Cachoeira, Estado do amazonas, Brasil. Rev PanAmaz Saúde. 2014;5(2):53-6.] URL: < http://scielo.iec.gov.br/scielo.php?script=sci_abs tract\&pid=S2176-

$62232014000200007 \& \operatorname{lng}=e s \& n r m=i s o \& t \operatorname{lng}=p t>$ 16. Silva D, Freire EPS. Pênfigo Foliáceo no Estado do Pará: estudo dos primeiros casos. R. Ci Bio. 1965;3: 15-25.

17. Talhari S, Fernandes G, Alecrin WD. Pênfigo foliáceo sul-americano no Estado do
Amazonas. Estudo de 10 casos. An Bras Dermatol. 1975;50: 49-52.

18. Silva $D$, Brito AC. Incidência dos pênfigos e dermatite de Duhring-Brocq no Pará. An Bras Dermatol. 1971;46: 31-4

19. Kridin K, Zelber-Sagi S, Bergman R. Pemphigus vulgaris and pemphigus foliaceus: differences in epidemiology and mortality. Acta Derm Venereol. 2017;97:1-5. URL: < https://pubmed.ncbi.nlm.nih.gov/28536732/>

20. Langan SM, Smeeth L, Hubbard R, Fleming KM, Smith CJ. West J. Bullouspemphigoid and pemphigus vulgaris-incidence and mortality in the UK: population based cohort study. BMJ 2008; 337: a180. URL: < https://www.bmj.com/content/337/bmj.a180>

21. Cunha PR, Barraviera SRCS. Dermatoses bolhosas autoimunes. An Bras Dermatol. 2009;84(2):111-24López IBH, Tarragó JM. Pénfigo vulgar - criterios actuals. Rev. Haban Cienc Méd. 2009;8(5):45-51. URL: < https://www.scielo.br/pdf/abd/v84n2/v84n2a03. pdf>

22. Bezerra OMPA, Galvão MAM, Silva DJ, Brito CRL, Rossini MCS, Gonçalves PMS et al. Pênfigo foliáceo endêmico (fogo selvagem) e sua associação com fatores ambientais e ocupacionais em Ouro Preto, Minas Gerais, Brasil. Cad. Saúde Colet. 2017; 25(2):225-32. URL: < https://www.scielo.br/scielo.php?pid=S1414$462 \times 2017005002106 \&$ script=sci_abstract \&tIng $=p$ $\mathrm{t}>$

23. Han A, Tan J, Bhambri DOS, Zeichner JA. A practical approach to treating autoimmune bullous disorders with systemic medications. J. Clin Aesthet Dermatol. 2009 ;2(5):19-28. URL: < https://www.ncbi.nlm.nih.gov/pmc/articles/PMC 2924135/>

24. Huang A, Madan RK. Future therapies for pemphigus vulgaris: rituximab and beyond. JAAD 2016;74(4):746-53. URL: < https://pubmed.ncbi.nlm.nih.gov/26792592/>

25. Bressan AL, Silva RS, Fontenelle E, Gripp AC. Imunossupressores na dermatologia. An. Bras. Dermatol. 2010;85(1):9-22. URL: < http://www.scielo.br/scielo.php?script=sci_artte $x t \& p i d=S 0365-05962010000100002>$

26. Pereira ALC, Bolzani FCB, Stefani $M$, Charlin R. Uso sistêmico de corticosteroides: revisão de literatura. Med. Cutan. Iber. Lat. Am. 2007;35(1):35-50. URL: < https://pesquisa.bvsalud.org/portal/resource/pt/ ibc-053909>

27. Aráuz Tinoco T. Comportamiento clínico epidemiológico en los pacientes diagnosticados con Pénfigo en el Centro Nacional de Dermatología 'Dr. Francisco José Gómez Urcuyo' 
en el período enero 1999 - diciembre 2003.

[tesis]. Managua: Universidad Nacional Autónoma de Nicaragua; 2004. URL: https://pesquisa.bvsalud.org/portal/resource/pt/ lil-399240>

28. Borges CB. Perfil evolutivo dos casos novos de pênfigo foliáceo endêmico ou fogo selvagem atendidos no hospital de referência do Estado de Goiás, HDT/AA. 1987-2006. [Dissertação - Mestrado]. Goiânia (GO): Universidade Federal de Goiás, 2008. URL: < https://files.cercomp.ufg.br/weby/up/59/o/Cami llaBorges-2008.pdf>

\section{Endereço para Correspondência}

Marcela Coutinho Alves

Tv Mercedes, no 208, Bairro São Bras.

Belém-Pará

CEP: $44031-460$

E-mail: marcelacalves95@gmail.com

Recebido em 19/11/2020

Aprovado em 01/05/2021

Publicado em 15/06/2021 